\title{
Inelastic neutron scattering study of undeuterated $\left[\mathrm{Mn}_{9} \mathrm{O}_{7}(\mathrm{OAc})_{11}(\right.$ thme $\left.)(\mathrm{py})_{3}\left(\mathrm{H}_{2} \mathrm{O}\right)_{2}\right]$
}

\author{
Roland Bircher ${ }^{\mathrm{a}, *}$, Grégory Chaboussant ${ }^{\mathrm{b}}$, Stefan T. Ochsenbein ${ }^{\mathrm{a}}$, \\ Felix Fernandez-Alonso ${ }^{c}$, Hans U. Güdel ${ }^{\text {a }}$, Euan K. Brechin ${ }^{*, d}$ \\ a Department of Chemistry and Biochemistry, University of Bern, Freiestrasse 3, CH-3000 Bern 9, Switzerland \\ ${ }^{\mathrm{b}}$ Laboratoire Léon Brillouin, CEA Saclay 91191 Gif-sur-Yvette Cedex, France \\ c ISIS Department, Rutherford Appleton Laboratory, Chilton, Didcot OX11 OQX England, UK \\ ${ }^{\mathrm{d}}$ School of Chemistry, The University of Edinburgh, West Mains Road, Edinburgh, EH9 3 JJ Scotland, UK
}

Received 5 October 2004; accepted 1 December 2004

Available online 25 May 2005

\begin{abstract}
We report on the magnetic properties of the enneanuclear mixed-valent single-molecule magnet $\left[\mathrm{Mn}_{9} \mathrm{O}_{7}(\mathrm{OAc})_{11}(\mathrm{thme})\right.$ (py) $)_{3}\left(\mathrm{H}_{2} \mathrm{O}\right)_{2}$ ] (1). Using inelastic neutron scattering (INS) spectroscopy, we have confirmed the $S=17 / 2$ cluster ground state of 1. Furthermore, we have determined accurately the anisotropy parameters of the zero field split ground state. The cluster 1 exhibits a strong negative axial anisotropy $D\left[\hat{S}_{z}^{2}-S(S+1) / 3\right]$ where $D=-0.249(5) \mathrm{cm}^{-1}$. It is necessary to include an axial higher order term $\left(B_{4}^{0}\right)$ in the effective spin Hamiltonian to account for the experimental data.
\end{abstract}

(C) 2005 Elsevier Ltd. All rights reserved.

Keywords: Single-molecule magnets; Inelastic neutron scattering; Magnetic anisotropy; Manganese

\section{Introduction}

Polymetallic cluster complexes of paramagnetic transition metal ions have attracted intense study since the discovery that such molecules can display the phenomenon of single-molecule magnetism (SMM) [1]. There is a barrier to relaxation of magnetisation in SMMs due to the combination of a large ground state spin multiplicity and a significant negative zero field splitting of that ground state. This imparts a molecular magnetic memory effect that can be observed as out-of-phase ac susceptibility signals or as hysteresis loops in $M$ versus $H$ studies. The last 10 years has seen many attempts to produce new SMMs in the same mould as the original

\footnotetext{
* Corresponding author. Tel.: +41316314254; fax: +41316314399 .

E-mail address: bircher@iac.unibe.ch (R. Bircher).
}

$\mathrm{SMM} \quad\left[\mathrm{Mn}_{12} \mathrm{O}_{12}(\mathrm{OAc})_{16}\left(\mathrm{H}_{2} \mathrm{O}\right)_{4}\right] \cdot 4 \mathrm{H}_{2} \mathrm{O} \cdot 2 \mathrm{CH}_{3} \mathrm{CO}_{2} \mathrm{H}$ and has led to the discovery of numerous SMMs ranging in nuclearity from 2 to $84[2,3]$. In most cases, the clusters have been made, at least initially, via the serendipitous assembly of metal salts with flexible organic bridging ligands such as carboxylates or alkoxides, and the majority have been clusters containing $\mathrm{Mn}^{\text {III }}$ ions.

In this article, we report an inelastic neutron scattering (INS) study of the mixed valent $\mathrm{SMM}\left[\mathrm{Mn}_{9} \mathrm{O}_{7^{-}}\right.$ $\left.(\mathrm{OAc})_{11}(\mathrm{thme})(\mathrm{py})_{3}\left(\mathrm{H}_{2} \mathrm{O}\right)_{2}\right]$ (1), which consists of a $\left[\mathrm{Mn}_{4}^{\text {III }} \mathrm{Mn}_{2}^{\text {II }} \mathrm{O}_{6}\right]$ ring on which is sitting a smaller $\left[\mathrm{Mn}_{3}^{\mathrm{IV}} \mathrm{O}\right]$ ring [4]. INS is a powerful tool to determine exchange couplings and single-ion anisotropies in molecular magnets [5]. Due to the spin of the neutron, there is a strong interaction between the magnetic moment of the neutron and the magnetic field created by the unpaired electrons in the sample. Measuring the INS intensity 
as a function of momentum and energy transfer provides useful information on the magnetic scattering function $S(Q, \omega)$, which contains the details about the magnetic exchange interactions and anisotropy splittings [6]. The focus of the present study is aimed at the determination of the anisotropy splitting of the ground state of $\mathbf{1}$.

To date no experimental study has provided direct information on the nature and energies of energy splitting in the ground state of $\mathbf{1}$. As a first approximation a giant spin model can be used to describe the ground state, assuming that it is well isolated from the excited spin states. An effective Hamiltonian then describes the zero field splitting of the giant spin ground state. The low $C_{1}$ symmetry of $\mathbf{1}$ allows for many higher order crystal field terms in the effective spin Hamiltonian. The fact that the Jahn-Teller axes of the four $\mathrm{Mn}^{\mathrm{III}}$ ions all lie roughly perpendicular to the plane of the $\left[\mathrm{Mn}_{4}^{\mathrm{III}} \mathrm{Mn}_{2}^{\mathrm{II}} \mathrm{O}_{6}\right]$ ring justifies the use of an axial Hamiltonian in first order

$H_{\text {axial }}=D\left[\hat{S}_{z}^{2}-S(S+1) / 3\right]+B_{4}^{0} \hat{O}_{4}^{0}$

with $\hat{O}_{4}^{0}=35 \hat{S}_{z}^{4}-[30 S(S+1)-25] \hat{S}_{z}^{2}-6 S(S+1)+3 S^{2}$ $(S+1)^{2}$. The first term of (1) splits the ground state in $S+1 / 2$ Kramer's doublets. For a negative $D$ value the $M_{S}= \pm S$ doublet lies lowest in energy.

A ground state spin of $S=17 / 2$ has been postulated for 1 on the basis of magnetic powder measurements [4]. Eq. (1) splits this ground state into nine $\pm M_{S}$ Kramer's doublets. An alternative solution with $S=19 / 2$ has also been postulated, which would give rise to 10 Kramer's doublets [4]. The INS selection rule $\Delta M_{S}= \pm 1$ predicts eight or nine transitions for an $S=17 / 2$ or $S=19 / 2$ ground state, respectively. The intensity for an $\left|M_{S}\right| \rightarrow\left|M_{S}+1\right|$ INS transition can be calculated as [7]

$$
\begin{aligned}
I\left(\left|M_{S}\right| \rightarrow\left|M_{S}+1\right|\right) \propto & P\left( \pm M_{S}\right) \cdot\left(\begin{array}{ccc}
S & 1 & S \\
-M_{S} & 1 & M_{S}+1
\end{array}\right) \\
& \cdot\left(\begin{array}{ccc}
S & 1 & S \\
-M_{S}-1 & 1 & M_{S}
\end{array}\right)
\end{aligned}
$$

with $P\left( \pm M_{S}\right)$ being the Boltzmann population of the initial state $\pm M_{S}$. The brackets are 3-j symbols.

\section{Experimental}

\subsection{Synthesis}

$\left[\mathrm{Mn}_{3} \mathrm{O}(\mathrm{OAc})_{6}(\text { py })_{3}\right] \cdot$ py was dissolved in $\mathrm{MeCN}$ with 1 equivalent of the ligand 1,1,1-tris(hydroxymethyl)ethane $\left(\mathrm{H}_{3}\right.$ thme $)$ and stirred for $12 \mathrm{~h}$. The solution was then filtered and Et2O added slowly. Crystals of $\left[\mathrm{Mn}_{9} \mathrm{O}_{7^{-}}\right.$ $(\mathrm{OAc})_{11}($ thme $\left.)(\mathrm{py})_{3}\left(\mathrm{H}_{2} \mathrm{O}\right)_{2}\right]$ (1) were obtained after 1 week.

\subsection{Physical measurements}

The INS experiments were performed on the inverted geometry time-of-flight spectrometer IRIS at the pulsed neutron spallation source ISIS at the Rutherford Appleton Laboratory, UK, using the PG002 graphite analyser with an analysing energy of $1.84 \mathrm{meV}$. Data were collected at three temperatures $1.6,12$, and $20 \mathrm{~K}$ and corrected for the background and detector efficiency by means of empty cell measurements and a vanadium reference. The time-of-flight to energy conversion and the data reduction were done using the program GUIDE. The instrumental resolution derived from a vanadium metal reference at the elastic position was $18 \mathrm{eV}$. On IRIS, the $\mathrm{ZnS}$ detector banks cover the angular range $2 \theta=20-160^{\circ}$ giving access to a momentum transfer range $Q=0.3-1.8 \AA^{-1}$ at the elastic position.

A $2.5 \mathrm{~g}$ fresh polycrystalline sample of undeuterated $\left[\mathrm{Mn}_{9} \mathrm{O}_{7}(\mathrm{OAc})_{11}(\right.$ thme $\left.)(\mathrm{py})_{3}\left(\mathrm{H}_{2} \mathrm{O}\right)_{2}\right]$ was used. The sample was placed under Helium in an aluminum hollow cylinder can with an outer diameter of $23 \mathrm{~mm}$ and a sample thickness of $2 \mathrm{~mm}$. The container was inserted in a standard ILL orange cryostat.

\section{Results and discussion}

Fig. 1 shows the INS spectra of 1 measured at $1.6 \mathrm{~K}$ $(10 \mathrm{~h}), 12 \mathrm{~K}(31 \mathrm{~h})$ and $20 \mathrm{~K}(25 \mathrm{~h})$, summed over all scattering angles. Positive and negative energy transfer corresponds to neutron energy loss and gain, respectively. The $1.6 \mathrm{~K}$ spectrum exhibits one strong band at $3.72 \mathrm{~cm}^{-1}$ on the loss side with a second one coming up as a shoulder at lower energy. A series of hot bands is observed at 12 and $20 \mathrm{~K}$. At this latter temperature,

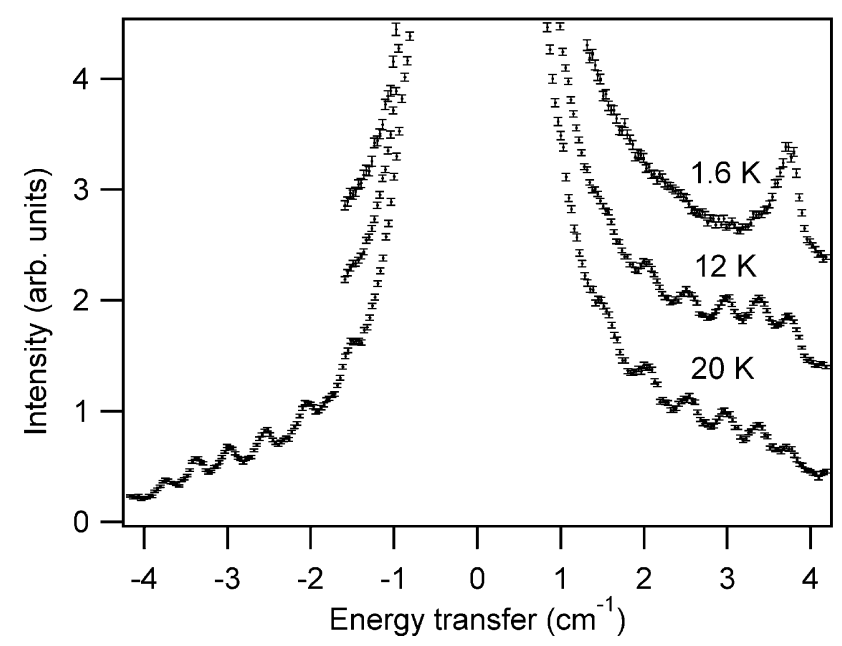

Fig. 1. INS spectra measured on IRIS with an analysing energy of $1.84 \mathrm{meV}$ at $1.6 \mathrm{~K}(10 \mathrm{~h}), 12 \mathrm{~K}(31 \mathrm{~h})$ and $20 \mathrm{~K}(25 \mathrm{~h})$, summed over all scattering angles. 
we measured the energy gain and the loss side. The peak energies are the same on both sides, and we can identify six distinct maxima and a shoulder on the elastic line on either side of the spectrum. The peaks exhibit an almost regular picket fence pattern with decreasing peak separations towards higher energy transfer. On the basis of their temperature and Q-dependence it is possible to assign the 14 observed bands to $\Delta M_{S}= \pm 1$ transitions within the ground state multiplet. The progression of the peak positions is limiting the possible number of transitions in the ground state multiplet to exactly eight on either side. Therefore, we can clearly rule out the possibility of an $S=19 / 2$ ground state. Our data unambiguously confirms the $S=17 / 2$ ground state.

Fig. 2(a) shows the spectrum measured at $20 \mathrm{~K}$ after subtracting the non-magnetic background. The peaks labelled I-VII correspond to the transitions shown in Fig. 3, which shows the energy splitting pattern of the $S=17 / 2$ derived from our data. Least-squares calculations using Eq. (1) and the peak positions determined by INS yield the following best parameter set: $D=-0.249(5) \mathrm{cm}^{-1}, B_{4}^{0}=7(4) \times 10^{-6} \mathrm{~cm}^{-1}$. The value

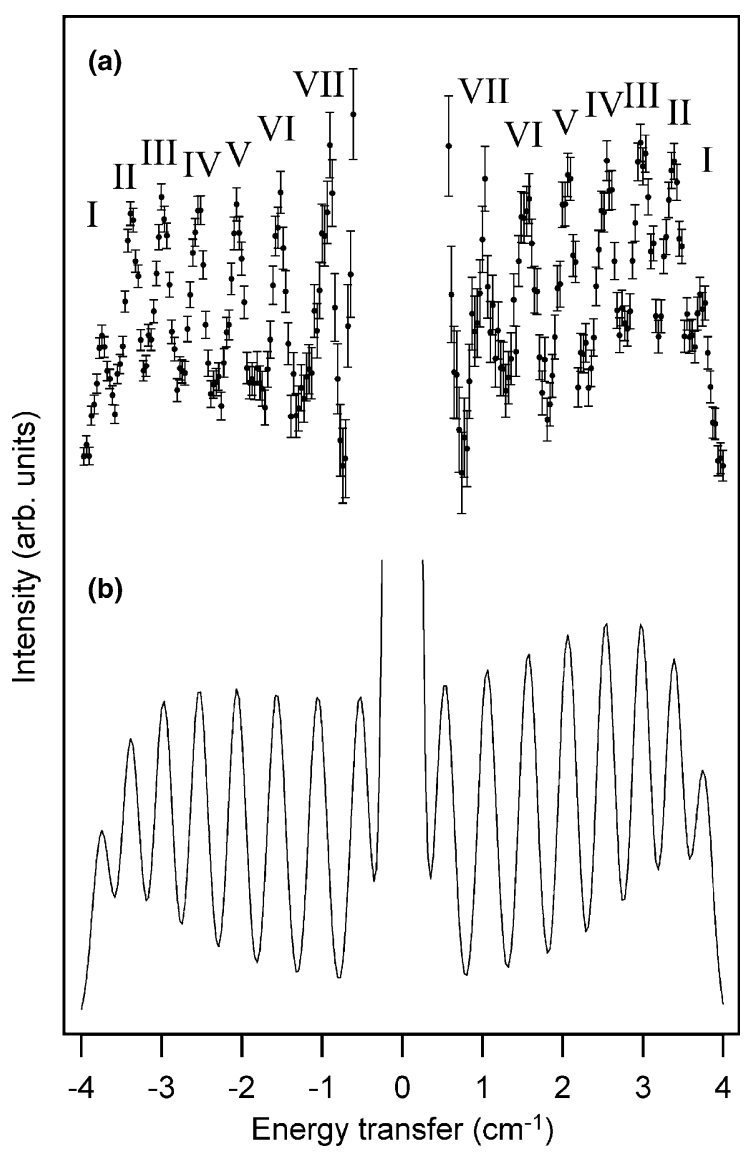

Fig. 2. (a) INS spectrum of $\mathrm{Mn}_{9}$ measured at $20 \mathrm{~K}$ and corrected for background. (b) Calculated spectrum at $20 \mathrm{~K}$ using Eqs. (1) and (2) with the parameters $D=-0.249 \mathrm{~cm}^{-1}$ and $B_{4}^{0}=7 \times 10^{-6} \mathrm{~cm}^{-1}$. The labelling of the peaks corresponds to the transitions in Fig. 3.

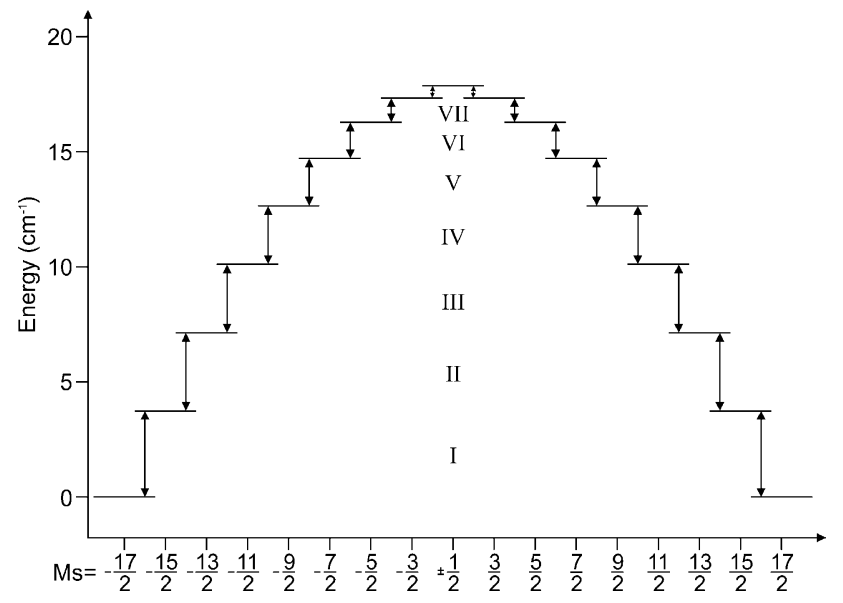

Fig. 3. Calculated anisotropy splitting of the $S=17 / 2$ ground state according to the Hamiltonian of Eq. (1) with parameter values $D=-0.249 \mathrm{~cm}^{-1}$ and $B_{4}^{0}=7 \times 10^{-6} \mathrm{~cm}^{-1}$ The double arrows correspond to the allowed $\Delta M_{S}= \pm 1$ INS transitions.

of the axial anisotropy parameter $D$ is in good agreement with the $D$ values of -0.29 and $-0.25 \mathrm{~cm}^{-1}$ derived from powder and single crystal magnetic measurements, respectively [8]. A Hamiltonian without the fourth order term $B_{4}^{0} \hat{O}_{4}^{0}$ could not account for the observed decrease of the energy intervals with increasing energy transfer. The $B_{4}^{0}$ parameter must be positive, which is not without precedent in the spin cluster literature. Positive $B_{4}^{0}$ values have been reported for example for the classical single molecule magnet $\mathrm{Fe}_{8}$ and for a $\mathrm{Mn}_{4}$ butterfly single molecule magnet $[9,10]$.

Fig. 2(b) shows a calculated spectrum at $20 \mathrm{~K}$ using Eqs. (1) and (2) with the best fit parameters. The peak positions and relative intensities are in excellent agreement with the experimental data shown in Fig. 2(a). For energy transfers below $\pm 1 \mathrm{~cm}^{-1}$ the experimental data are dominated by the elastic line, and the eighth peak predicted by the calculations is not observable. The non-magnetic scattering is orders of magnitude more intense than the magnetic scattering in the region of the elastic line, thus swamping the magnetic part.

Physically, the strong central line with the pronounced tails in Fig. 1 originates from elastic and quasielastic non-magnetic scattering processes. Its intensity is enhanced by the presence of a large number of hydrogen atoms in the measured sample. Hydrogen is a strong incoherent nuclear scatterer. This is exploited in molecular vibrational spectroscopy [11]. At the low energy transfer of our experiment, it leads to an enhanced elastic line including tails as well as a higher background. For a more accurate determination of the peak VII in Fig. 2(a), it would be necessary to measure a deuterated sample. The incoherent nuclear scattering cross-section of deuterium is 40 times smaller than the one of hydrogen; therefore, the effects described above are reduced 
drastically. Deuteration is often considered a prerequisite for a successful magnetic INS experiment, but the present study shows that data of remarkable quality can be obtained with an undeuterated sample in the energy transfer range between 1 and $4 \mathrm{~cm}^{-1}$ also. We have recently studied a number of undeuterated molecular spin clusters by INS and made similar observations $[12,13]$. The energy transfer region, in which magnetic cluster excitations can be observed despite the presence of hydrogen atoms, covers the range from about 1 to $30 \mathrm{~cm}^{-1}$. We ascribe this to a low density of vibrational states with significant hydrogen contribution in this "lucky window".

\section{Conclusions}

INS measurements unambiguously prove the $S=17 / 2$ ground state of $\mathbf{1}$. Analysis of the INS transitions within the zero field split ground state lead to a very accurate determination of the axial anisotropy parameter $D$ and the crystal field parameter $B_{4}^{0}$. The experimental INS results of this study are of remarkable quality, despite the presence of a large number of hydrogen atoms in the sample.

\section{References}

[1] G. Christou, D. Gatteschi, D.N. Hendrickson, R. Sessoli, MRS Bull. 25 (2000) 66.

[2] H. Miyasaka, R. Clérac, W. Wernsdorfer, L. Lecren, C. Bonhomme, K.-I. Sugiura, M. Yamashita, Angew. Chem., Int. Ed. 43 (2004) 2801.

[3] A.J. Tasiopoulos, A. Vinslava, W. Werndorfer, K.A. Abboud, G. Christou, Angew. Chem., Int. Ed. 43 (2004) 2117.

[4] E.K. Brechin, M. Soler, J. Davidson, D.N. Hendrickson, S. Parsons, G. Christou, Chem. Commun. (2002) 2252.

[5] R. Basler, C. Boskovic, G. Chaboussant, H.U. Güdel, M. Murrie, S.T. Ochsenbein, A. Sieber, Chem. Phys. Chem. 4 (2003) 910.

[6] P. Böni, A. Furrer, J. Schefer, Principles of neutron scattering, in: Proceedings of the Eighth Summer School on Neutron Scattering, World Scientific, Singapore, 2000.

[7] J.R. Birgeneau, J. Phys. Chem. Solids 33 (1972) 59.

[8] E.K. Brechin, M. Soler, G. Christou, J. Davidson, D.N. Hendrickson, S. Parsons, W. Wernsdorfer, Polyhedron 22 (2003) 1771.

[9] R. Caciuffo, G. Amoretti, A. Murani, R. Sessoli, A. Caneschi, D. Gatteschi, Phys. Rev. Lett. 81 (1998) 4744

[10] J. Yoo, A. Yamaguchi, M. Nakano, J. Krzystek, W.E. Streib, L.-C. Brunel, H. Ishimoto, G. Christou, D.N. Hendrickson, Inorg. Chem. 40 (2001) 4604

[11] G.F. Longster, J.W. White, Mol. Phy. 17 (1969) 1.

[12] H. Andres, R. Basler, H.U. Güdel, G. Aromi, G. Christou, H. Büttner, B. Ruffle, J. Am. Chem. Soc. 122 (2000) 12469.

[13] C. Boskovic, A. Sieber, G. Chaboussant, H.U. Güdel, J. Ensling, W. Wernsdorfer, A. Neels, G. Labat, H. Stoeckli-Evans, S. Janssen, Inorg. Chem. 43 (2004) 5053. 